Département de sciences économiques

2007-02

Free Triples, Large Indifference Classes and the Majority Rule

EHLERS, Lars

BARBERÀ, Salvador 


\section{Département de sciences économiques}

Université de Montréal

Faculté des arts et des sciences

C.P. 6128, succursale Centre-Ville

Montréal (Québec) H3C 3J7

Canada

http://www.sceco.umontreal.ca

SCECO-information@UMontreal.CA

Téléphone : (514) 343-6539

Télécopieur : (514) 343-7221

Ce cahier a également été publié par le Centre interuniversitaire de recherche en économie quantitative (CIREQ) sous le numéro 02-2007.

This working paper was also published by the Center for Interuniversity Research in Quantitative Economics (CIREQ), under number 02-2007.

ISSN 0709-9231 


\title{
Free Triples, Large Indifference Classes and the Majority Rule*
}

\author{
Salvador Barberà ${ }^{\dagger} \quad$ Lars Ehlers ${ }^{\ddagger}$
}

March 2007

\begin{abstract}
We consider situations in which agents are not able to completely distinguish between all alternatives. Preferences respect individual objective indifferences if any two alternatives are indifferent whenever an agent cannot distinguish between them. We present necessary and sufficient conditions of such a domain of preferences under which majority rule is quasi-transitive and thus Condorcet winners exist for any set of alternatives. Finally, we compare our proposed restrictions with others in the literature, to conclude that they are independent of any previously discussed domain restriction.

JEL classification: C71, D71.

Keywords: Quasi-Transitivity, Majority Rule.
\end{abstract}

\footnotetext{
${ }^{*}$ We acknowledge useful comments and suggestions by Gabrielle Demange and Matt Jackson. Barberà acknowledges financial support from the Barcelona Economics Program (CREA), the Spanish Ministry of Education and Science through grant Acciones Complementarias SEJ2006-27589-E and FEDER, and the Generalitat de Catalunya, Departament d'Universitats, Recerca i Societat de la Informaci through grant SGR2000-00054. Part of this paper was written while the second author was a Marie Curie Research Fellow at C.O.D.E. at the Universitat Autònoma de Barcelona. He acknowledges the hospitality of the Departament d'Economia i d'Història Econòmica and financial support from the European Union under Grant No. HPMT-CT-2000-0017.

${ }^{\dagger}$ Departament d'Economia i d'Història Econòmica and C.O.D.E., Universitat Autònoma de Barcelona, 08193 Bellaterra, Barcelona, Spain, e-mail: salvador. barbera@uab.es.

‡Département de Sciences Économiques and CIREQ, Université de Montréal, Montréal, Québec H3C 3J7, Canada, e-mail: lars.ehlers@umontreal.ca.
} 


\section{Introduction}

When societies ask their members to vote, different voters have reason to view different alternatives under different lights. Some may care a lot for some of the issues, and not for others. Some may be aware of differences that others can hardly perceive. Some may be strongly affected by measures which are irrelevant for the life of others. Differences in opinion, information and interest are only three of the reasons why not everyone attaches the same importance to all of the choices faced by society. This is the reason why we want to model classes of voting situations where the sets of preferences which are admissible for some voter are different from those that one can expect from others. More specifically, we emphasize the asymmetries in individual preferences that result from the fact that some voters may systematically rank some alternatives as being indifferent, while other voters may be able to sharply distinguish between them, and to express strong preferences in their regard.

Specifically, we shall consider preference domains where each agent is characterized by a partition of the set of alternatives. Each set in the partition of an agent includes those alternatives that will always be indifferent to each other, from the viewpoint of this voter. Indifference stands here to express a number of circumstances that could be differentiated in other contexts, but that become equivalent for our analysis of the majority rule. Two alternatives will be treated as necessarily indifferent if it is the case that the agent will never prefer one above the other, whether this is due to lack of interest, opinion or information.

Although details will be provided later, let us emphasize that we are not characterizing a specific preference domain, but a family of such. Each of the domains in the family will be specified once a partition of the set of alternatives is attributed to each voter, satisfying for each triple of alternatives one of two joint conditions on the partition. Roughly speaking, these conditions express the requirement that the classes of alternatives for which we allow voters to be indifferent must be interrelated among each other. Any triple of alternatives may be "free" for some agents, admitting the ranking of the three by these voters in any order. However, the required interrelations among partitions of alternatives for different agents must be such that, eventually, the existence of these "free triples" does not disrupt the effectiveness of majority voting.

Our main result is that any partition satisfying our conditions defines a family of preference profiles under which majority rule is always quasitransitive. Therefore, we characterize a family of domain restrictions, based on the distribution of indifferences across members of society, under which 
one can guarantee that Condorcet winners always exist. Since our condition defines a wide variety of possible environments, we hope that authors may find the opportunity to specialize their models in a variety of ways that fit our general pattern. But we are already in position to suggest a number of applications.

For a first example, consider an election in which the candidates are linearly ordered, from left to right. Any agent is eligible as a candidate, and is entitled to decline the position if eventually elected. It is therefore interesting to establish not only a winner, but also a list of alternates in case of resignation. Each agent has then one neighbor to his left, and one to his right (except for those at the extremes, who only have one neighbor). We allow each agent to freely order himself and its immediate neighbors. Some agent may prefer to be elected rather than seeing his neighbors elected. Others may prefer their neighbor to the right (or left) to be the winner. Likewise, second and third positions in the ranking are free. Hence, our preferences allow for free triples of alternatives. Yet, we also assume that agents cannot clearly distinguish between a victory by their neighbor in the right and the victory of any other candidate in the same direction (this may be due to myopia, or else result from rational calculations). He is indifferent among all candidates to his right, and also among all candidates to his left. In this example, then, each agent has (at most) three indifference classes: all people to the left of the voter form a class, all people to the right of the voter form a second class, and the voter himself is a third class. These classes can be ordered in any possible way. We shall prove that if all agents have preferences of this type, the majority relation associated to any profile of opinions by any number of voters is quasi-transitive (i.e., the strict majorities among alternatives respect transitivity). This is sufficient for the existence of Condorcet winners. It also allows to guarantee the existence of path independent selections (Plott, 1973) from the set of Condorcet winners: elections can be organized sequentially without any fear for agenda manipulation.

Our example can be extended. The linear structure is unnecessarily narrow. Agents may be at the nodes of any tree. Therefore, each agent may have a different number of neighbors, as many as the number of branches starting from or arriving to that node. An agent with $k$ neighbors may now freely rank $k+1$ classes of alternatives, including oneself as a singleton class. Each of the remaining classes includes one neighbor and all other candidates which are further out than this neighbor within the tree structure. This is a very substantial extension of the domain: very perceptive people can have many neighbors and thus freely rank many classes of candidates. Other agents may be restricted by their positions to only rank a few groups. Many structures are allowed, provided they can be represented by a tree, of any 
form. We later go further into the example based on a tree by connecting it with a model recently discussed by Demange (2004).

These examples are rather special, and leave room for many other applications. Yet, the examples already suggest that the reasons for agents to be indifferent among sets of alternatives may come from different sources, ranging from technological to purely subjective, and may be associated to asymmetries of different sorts, which can be formalized through networks, rankings or partitions. We are aware that in certain contexts it may be attractive to distinguish between indifference and incompleteness when modelling the inability or the lack of willingness of some agents to express a strict preference between some pairs of alternatives. But we feel that this distinction would not lead to any substantial or operational gain in our context, since expressing indifference or not expressing any preference are equivalent for the workings of majority rule.

The paper proceeds as follows. In Section 2, we present our model. In Section 3, we state and prove the main result, and we show that the examples we have briefly presented are indeed instances where our condition holds. In Section 4, we discuss the differences between our suggested domain restrictions and those previously considered by the literature on social choice and political economy.

\section{Agents and Preferences}

Let $N=\{1, \ldots, n\}$ denote the finite set of agents and $A$ the finite set of alternatives. We always assume that $|N| \geq 3$ and $|A| \geq 3$. Agents have preferences on the set of alternatives, which are given by complete, reflexive, and transitive binary relations on $A$. The preference relation of agent $i$ is denoted by $R_{i}$, his strict preference relation by $P_{i}$ and his indifference relation by $I_{i}$. Let $\mathcal{R}$ denote the set of all complete, reflexive, and transitive binary relations on $A$. A (preference) profile is a list $R=\left(R_{i}\right)_{i \in N}$ specifying for each agent $i$ his preference relation $R_{i}$ on $A$. Let $\mathcal{R}^{N}$ denote the set of all profiles.

We consider the problem of society $N$ having to choose a member from $A$. For example, $N$ is a committee or a faculty board having to elect a chair from $A$. In such a situation an agent often does not have sufficient information to distinguish between all members of $A$. In other words his preference relation will express objective indifferences, i.e., he views indifferent any two alternatives which he cannot distinguish. The objective indifferences of agent $i$ are given by a partition $\mathcal{S}_{i}$ of $A$. Then $\mathcal{R}_{\mathcal{S}_{i}}$ is the set of all preference relations respecting $i$ 's objective indifferences: for all $R_{i} \in \mathcal{R}$ :

$$
R_{i} \in \mathcal{R}_{\mathcal{S}_{i}} \Leftrightarrow a I_{i} b \text { for all } a, b \in A \text { such that }\{a, b\} \subseteq S \text { for some } S \in \mathcal{S}_{i} .
$$


Note that the domain $\mathcal{R}_{\mathcal{S}_{i}}$ does not exclude any subjective indifferences of $i$ (given his objective indifferences). We may want to rule out any indifferences between alternatives belonging to different members of $\mathcal{S}_{i}$ since agent $i$ is able to distinguish them. In other words, agent $i$ 's preference will often be strict for alternatives belonging to different members of $\mathcal{S}_{i}$. Then $\mathcal{P}_{\mathcal{S}_{i}}$ is the set of all preference relations respecting $i$ 's objective indifferences and being strict for any two alternatives which agent $i$ is able to distinguish: for all $R_{i} \in \mathcal{R}$ :

$R_{i} \in \mathcal{P}_{\mathcal{S}_{i}} \Leftrightarrow R_{i} \in \mathcal{R}_{\mathcal{S}_{i}} \& a P_{i} b \vee b P_{i} a$ for all $a, b \in A$ such that $\{a, b\} \nsubseteq S$ for all $S \in \mathcal{S}_{i}$.

Let $\mathcal{S}=\left(\mathcal{S}_{i}\right)_{i \in N}$ denote the profile of individual partitions of $A, \mathcal{R}_{\mathcal{S}}=$ $\times_{i \in N} \mathcal{R}_{\mathcal{S}_{i}}$, and $\mathcal{P}_{\mathcal{S}}=\times_{i \in N} \mathcal{P}_{\mathcal{S}_{i}}$. We call $\mathcal{R}_{\mathcal{S}}$ the set of weak profiles which are admissible under $\mathcal{S}$ and $\mathcal{P}_{\mathcal{S}}$ the set of strict profiles which are admissible under $\mathcal{S}$. A triple (of alternatives) is a subset of $A$ containing exactly three elements. We call a triple $\{a, b, c\}$ free in $\mathcal{S}_{i}$ if $i$ is able to distinguish between $a, b$ and $c$, i.e., for all $S \in \mathcal{S}_{i},|S \cap\{a, b, c\}| \leq 1$ (and agent $i$ may rank the alternatives $a, b$, and $c$ in any conceivable way).

When $\mathcal{S}_{i}$ is the finest partition, $i$ can perfectly distinguish among all alternatives. Many papers start from the domain of linear orderings and use the results derived for the linear case for the whole set of weak orderings. We cannot use this approach here since linear orderings are often ruled out by $\mathcal{S}$.

\section{Quasi-Transitivity of the Majority Rule}

Majority is one possibility for society to choose one alternative from $A$. Our purpose is to identify necessary and sufficient conditions for quasi-transitivity of the majority rule given the agents' objective indifferences. For each profile the majority relation is defined as follows: given $R \in \mathcal{R}^{N}$ and $a, b \in A$, its majority (binary) relation, denoted by $R^{m}$, is defined as

$$
a R^{m} b \Leftrightarrow\left|\left\{i \in N \mid a P_{i} b\right\}\right| \geq\left|\left\{i \in N \mid b P_{i} a\right\}\right| .
$$

It is well known that the majority relation derived from an arbitrary preference profile may be cyclic. When this occurs, the choices by majority are not well defined. We are thus particularly interested in conditions guaranteeing that this problem is avoided. For each profile $R$, an alternative is a (Condorcet) winner if it is not beaten (in the strict sense) by another alternative under the majority relation, i.e., $a \in A$ is a (Condorcet) winner of $R$ if for all $b \in A, a R^{m} b$. The existence of winners is guaranteed at those profiles where the majority relation is quasi-transitive (i.e., the strict relation 
associated with the majority relation is transitive ${ }^{1}$ ). Formally, the majority relation $R^{m}$ is quasi-transitive if for all triples $\{a, b, c\}$ the following holds: $a P^{m} b \& b P^{m} c \Rightarrow a P^{m} c$.

Our basic result provides necessary and sufficient conditions for the quasitransitivity of majority rule on the set of strict profiles which are admissible under the objective indifferences $\mathcal{S}$.

Definition $1 l$ Dichotomous Preferences ( $l$ DP). The partition profile $\mathcal{S}$ satisfies $l$ DP for a triple $\{a, b, c\}$ if at least $l$ agents cannot distinguish two alternatives in the triple, i.e., there is $L \subseteq N$ such that $|L| \geq l$ and for each $i \in L$ there is some $S \in \mathcal{S}_{i}$ with $|S \cap\{a, b, c\}| \geq 2$.

Our next property requires the following notation. Fix the partition profile $\mathcal{S}$ and a triple $\{a, b, c\} \in A$. Let

$$
N_{[a b]}=\left\{i \in N \mid \text { for some } S \in \mathcal{S}_{i},\{a, b\} \subseteq S \text { and } c \notin S\right\}
$$

denote the agents who cannot distinguish between $a$ and $b$ and who distinguish $c$ from $a$ and $b$. Similarly we define $N_{[a c]}$ and $N_{[b c]}$. Also let $n_{[a b]}=\left|N_{[a b]}\right|, n_{[a c]}=\left|N_{[a c]}\right|$, and $n_{[b c]}=\left|N_{[b c]}\right|$. Abusing language, we will use the name "parity" to denote the property of a number being odd or even.

Definition 2 (n-2) Equal Parity Dichotomous Preferences ((n-2) EPDP). The partition profile $\mathcal{S}$ satisfies $(n-2)$ EPDP for a triple $\{a, b, c\}$ if it satisfies $(n-2)$ DP and the numbers of agents, who cannot distinguish exactly two alternatives in the triple, are either all even or all odd, i.e., the numbers $n_{[a b]}$, $n_{[a c]}$, and $n_{[b c]}$ are either all even or all odd.

Theorem 1 Let $\mathcal{S}=\left(\mathcal{S}_{i}\right)_{i \in N}$. Then majority rule is quasi-transitive on the domain $\mathcal{P}_{\mathcal{S}}$ if and only if $\mathcal{S}$ satisfies $(n-1)$ DP or $(n-2)$ EPDP for any triple of alternatives.

Proof. Throughout the proof we fix a triple $\{a, b, c\}$ of $A$. Let $N_{\{a, b, c\}}^{f}$ denote the set of agents for whom $\{a, b, c\}$ is free in $\mathcal{S}_{i}$, i.e., they may have any ranking on $\{a, b, c\}$. Formally,

$$
N_{\{a, b, c\}}^{f}=\left\{i \in N \mid \text { for all } S \in \mathcal{S}_{i},|S \cap\{a, b, c\}| \leq 1\right\} .
$$

Given a profile $R$ and $x, y \in A$, let $N_{x y}^{R}=\left\{i \in N \mid x P_{i} y\right\}$ denote the set of agents who strictly prefer $x$ to $y$ under $R$.

\footnotetext{
${ }^{1}$ We have $a P^{m} b$ in (1) if the inequality is strict.
} 
(Only if) We show necessity. Suppose that the majority rule is quasi-transitive on the domain $\mathcal{P}_{\mathcal{S}}$ and consider the triple $\{a, b, c\}$. We distinguish several cases depending on the number of agents for whom the triple $\{a, b, c\}$ is free.

Case 1: $\left|N_{\{a, b, c\}}^{f}\right| \leq 1$.

Then for all $i \in N \backslash N_{\{a, b, c\}}^{f}$ there is some $S \in \mathcal{S}_{i}$ such that

$$
(\{a, b\} \subseteq S) \vee(\{b, c\} \subseteq S) \vee(\{a, c\} \subseteq S) .
$$

Hence, $\mathcal{S}$ satisfies $(n-1)$ DP for $\{a, b, c\}$.

Case 2: $\left|N_{\{a, b, c\}}^{f}\right|=2$.

Suppose that $\mathcal{S}$ does not satisfy $(n-2) \operatorname{EPDP}$ for $\{a, b, c\}$. By $\left|N_{\{a, b, c\}}^{f}\right|=$ $2, \mathcal{S}$ satisfies $(n-2) \mathrm{DP}$ for $\{a, b, c\}$ and either exactly one or exactly two of the numbers $n_{[a b]}, n_{[a c]}$, and $n_{[b c]}$ are odd. Let $N_{\{a, b, c\}}^{f}=\{1,2\}$.

First, without loss of generality, suppose that $n_{[a c]}$ is odd and $n_{[a b]}$ and $n_{[b c]}$ are even. Let $i \in N_{[a c]}$. Note that $\left|N_{[a c]} \backslash\{i\}\right|$ is even. Then choose $R \in \mathcal{P}_{\mathcal{S}}$ such that

$$
\text { (1) } c P_{1} a P_{1} b ; \quad(2) a P_{2} b P_{2} c ; \quad \text { (3) } b P_{i} a I_{i} c
$$

half of the agents in $N_{[a c]} \backslash\{i\}$ strictly prefer $a$ and $c$ to $b$ and the other half of the agents in $N_{[a c]} \backslash\{i\}$ strictly prefer $b$ to $a$ and $c$; half of the agents in $N_{[a b]}$ strictly prefer $a$ and $b$ to $c$ and the other half of the agents in $N_{[a b]}$ strictly prefer $c$ to $a$ and $b$; and half of the agents in $N_{[b c]}$ strictly prefer $b$ and $c$ to $a$ and the other half of the agents in $N_{[b c]}$ strictly prefer $a$ to $b$ and c. Now by construction, $\left|N_{a b}^{R}\right|>\left|N_{b a}^{R}\right|$ and $a P^{m} b,\left|N_{b c}^{R}\right|>\left|N_{c b}^{R}\right|$ and $b P^{m} c$, and $\left|N_{a c}^{R}\right| \leq\left|N_{c a}^{R}\right|$ and $c R^{m} a$. Hence, majority rule is not quasi-transitive.

Second, without loss of generality, suppose that $n_{[a b]}$ and $n_{[b c]}$ are odd and $n_{[a c]}$ is even. Let $i \in N_{[a b]}$ and $j \in N_{[b c]}$. Note that $\left|N_{[a b]} \backslash\{i\}\right|$ and $\left|N_{[b c]} \backslash\{j\}\right|$ are even. Then choose $R \in \mathcal{P}_{\mathcal{S}}$ such that

$$
\text { (1) } c P_{1} a P_{1} b ; \quad \text { (2) } b P_{2} c P_{2} a ; \quad \text { (3) } a I_{i} b P_{i} c ; \quad \text { (4) } a P_{j} b I_{j} c \text {; }
$$

half of the agents in $N_{[a b]} \backslash\{i\}$ strictly prefer $a$ and $b$ to $c$ and the other half of the agents in $N_{[a b]} \backslash\{i\}$ strictly prefer $c$ to $a$ and $b$; half of the agents in $N_{[b c]} \backslash\{j\}$ strictly prefer $b$ and $c$ to $a$ and the other half of the agents in $N_{[b c]} \backslash\{j\}$ strictly prefer $a$ to $b$ and $c$; and half of the agents in $N_{[a c]}$ strictly prefer $a$ and $c$ to $b$ and the other half of the agents in $N_{[a c]}$ strictly prefer $b$ to $a$ and $c$. Now by construction, $\left|N_{a b}^{R}\right|>\left|N_{b a}^{R}\right|$ and $a P^{m} b,\left|N_{b c}^{R}\right|>\left|N_{c b}^{R}\right|$ and $b P^{m} c$, and $\left|N_{a c}^{R}\right| \leq\left|N_{c a}^{R}\right|$ and $c R^{m} a$. Hence, majority rule is not quasi-transitive.

Case 3: $\left|N_{\{a, b, c\}}^{f}\right|$ is even and $\left|N_{\{a, b, c\}}^{f}\right| \geq 4$. 
If either exactly one or exactly two of the numbers $n_{[a b]}, n_{[a c]}$, and $n_{[b c]}$ are odd, then we derive a contradiction identically as in Case 2: we endow one half of the agents in $N_{\{a, b, c\}}^{f}$ with the preference relation of agent 1 in Case 2 and the other half of the agents in $N_{\{a, b, c\}}^{f}$ with the preference relation of agent 2 in Case 2.

Otherwise, let $\left|N_{\{a, b, c\}}^{f}\right|=2+2 l$ and $\{1,2,3,4\} \subseteq N_{\{a, b, c\}}^{f}$. Let

$$
\text { (1) } c P_{1} a P_{1} b ; \quad \text { (2) } a P_{2} b P_{2} c ; \quad \text { (3) } a P_{3} b P_{3} c ; \quad \text { (4) } b P_{4} c P_{4} a \text {. }
$$

First, suppose that all the numbers $n_{[a b]}, n_{[a c]}$, and $n_{[b c]}$ are even. Then choose $R \in \mathcal{P}_{\mathcal{S}}$ such that (1)-(4) hold, $l$ agents (including 1 ) in $N_{\{a, b, c\}}^{f}$ have agent 1's preference relation, $l$ agents (including 4$)$ in $N_{\{a, b, c\}}^{f}$ have agent 4 's preference relation, half of the agents in $N_{[a b]}$ strictly prefer $a$ and $b$ to $c$ and the other half of the agents in $N_{[a b]}$ strictly prefer $c$ to $a$ and $b$; half of the agents in $N_{[b c]}$ strictly prefer $b$ and $c$ to $a$ and the other half of the agents in $N_{[b c]}$ strictly prefer $a$ to $b$ and $c$; and half of the agents in $N_{[a c]}$ strictly prefer $a$ and $c$ to $b$ and the other half of the agents in $N_{[a c]}$ strictly prefer $b$ to $a$ and c. Now by construction, $\left|N_{a b}^{R}\right|>\left|N_{b a}^{R}\right|$ and $a P^{m} b,\left|N_{b c}^{R}\right|>\left|N_{c b}^{R}\right|$ and $b P^{m} c$, and $\left|N_{a c}^{R}\right| \leq\left|N_{c a}^{R}\right|$ and $c R^{m} a$. Hence, majority rule is not quasi-transitive.

Second, suppose that all the numbers $n_{[a b]}, n_{[a c]}$, and $n_{[b c]}$ are odd. Let $i \in N_{[a b]}, j \in N_{[b c]}$, and $k \in N_{[a c]}$. Note that $\left|N_{[a b]} \backslash\{i\}\right|,\left|N_{[b c]} \backslash\{j\}\right|$, and $\left|N_{[a c]} \backslash\{k\}\right|$ are even. Then choose $R \in \mathcal{P}_{\mathcal{S}}$ such that (1)-(4) hold, $l$ agents (including 1) in $N_{\{a, b, c\}}^{f}$ have agent 1's preference relation, $l$ agents (including 4) in $N_{\{a, b, c\}}^{f}$ have agent 4 's preference relation,

$$
\text { (5) } c P_{i} a I_{i} b ; \quad \text { (6) } a P_{j} b I_{j} c ; \quad \text { (7) } b P_{k} a I_{k} c
$$

half of the agents in $N_{[a b]} \backslash\{i\}$ strictly prefer $a$ and $b$ to $c$ and the other half of the agents in $N_{[a b]} \backslash\{i\}$ strictly prefer $c$ to $a$ and $b$; half of the agents in $N_{[b c]} \backslash\{j\}$ strictly prefer $b$ and $c$ to $a$ and the other half of the agents in $N_{[b c]} \backslash\{j\}$ strictly prefer $a$ to $b$ and $c$; and half of the agents in $N_{[a c]} \backslash\{k\}$ strictly prefer $a$ and $c$ to $b$ and the other half of the agents in $N_{[a c]} \backslash\{k\}$ strictly prefer $b$ to $a$ and $c$. Now by (5), (6), and (7), the agents $i, j$, and $k$ offset each other for any binary comparison among $a, b$, and $c$. Thus, by construction, $\left|N_{a b}^{R}\right|>\left|N_{b a}^{R}\right|$ and $a P^{m} b,\left|N_{b c}^{R}\right|>\left|N_{c b}^{R}\right|$ and $b P^{m} c$, and $\left|N_{a c}^{R}\right| \leq\left|N_{c a}^{R}\right|$ and $c R^{m} a$. Hence, majority rule is not quasi-transitive.

Case 4: $\left|N_{\{a, b, c\}}^{f}\right|$ is odd and $\left|N_{\{a, b, c\}}^{f}\right| \geq 3$.

Let $\left|N_{\{a, b, c\}}^{f}\right|=3+2 l$ and $\{1,2,4\} \subseteq N_{\{a, b, c\}}^{f}$. Similarly as in Case 3, we endow agents 1,2 , and 4 with the same preferences as in Case $3, l$ agents 
in $N_{\{a, b, c\}}^{f} \backslash\{1,2,4\}$ with the preference relation of agent 1 , and the other $l$ agents in $N_{\{a, b, c\}}^{f} \backslash\{1,2,4\}$ with the preference relation of agent 4 .

If all the numbers $n_{[a b]}, n_{[a c]}$, and $n_{[b c]}$ have the same parity (they are either all odd or all even), then we obtain a contradiction similarly as in Case 3.

If exactly one of the numbers $n_{[a b]}, n_{[a c]}$, and $n_{[b c]}$ is odd, say $n_{[b c]}$, then choose $i \in N_{[b c]}$ and let $a P_{i} b I_{i} c$. Note that $\left|N_{[b c]} \backslash\{i\}\right|$ is even. Now similarly to Case 3 we can show that majority rule is not quasi-transitive.

If exactly two of the numbers $n_{[a b]}, n_{[a c]}$, and $n_{[b c]}$ are odd, say $n_{[b c]}$ and $n_{[a c]}$, then choose $i \in N_{[b c]}$ and $j \in N_{[a c]}$. Let $a P_{i} b I_{i} c$ and $b P_{j} a I_{j} c$. Note that $\left|N_{[b c]} \backslash\{i\}\right|$ and $\left|N_{[a c]} \backslash\{j\}\right|$ are even. Now similarly to Case 3 we can show that majority rule is not quasi-transitive.

(If) We show sufficiency. Let $R \in \mathcal{P}_{\mathcal{S}}$ be such that $a P^{m} b$ and $b P^{m} c$. We need to show $a P^{m} c$.

Note that for all $i \in N_{[a b]}, a I_{i} b P_{i} c$ or $c P_{i} a I_{i} b$. Thus, we have

$$
\left\{\begin{array}{l}
N_{a c}^{R} \cap N_{[a b]}=N_{b c}^{R} \cap N_{[a b]} \\
N_{c a}^{R} \cap N_{[a b]}=N_{c b}^{R} \cap N_{[a b]}
\end{array}\right\} .
$$

Let $k_{[a b]}=\left|N_{a c}^{R} \cap N_{[a b]}\right|$ and $k_{[a b]}^{\prime}=\left|N_{c a}^{R} \cap N_{[a b]}\right|$. Similarly,

$$
\left\{\begin{array}{l}
N_{a c}^{R} \cap N_{[b c]}=N_{a b}^{R} \cap N_{[b c]} \\
N_{c a}^{R} \cap N_{[b c]}=N_{b a}^{R} \cap N_{[b c]}
\end{array}\right\}
$$

Let $k_{[b c]}=\left|N_{a c}^{R} \cap N_{[b c]}\right|$ and $k_{[b c]}^{\prime}=\left|N_{c a}^{R} \cap N_{[b c]}\right|$. Again similarly,

$$
\left\{\begin{array}{l}
N_{a b}^{R} \cap N_{[a c]}=N_{c b}^{R} \cap N_{[a c]} \\
N_{b a}^{R} \cap N_{[a c]}=N_{b c}^{R} \cap N_{[a c]}
\end{array}\right\} .
$$

Let $k_{[a c]}=\left|N_{a b}^{R} \cap N_{[a c]}\right|$ and $k_{[a c]}^{\prime}=\left|N_{b a}^{R} \cap N_{[a c]}\right|$.

First, suppose that $\mathcal{S}$ satisfies $(n-1)$ DP for the triple $\{a, b, c\}$. Without loss of generality, let $N_{\{a, b, c\}}^{f}=\{1\}$ (the proof for the case $N_{\{a, b, c\}}^{f}=\emptyset$ follows the same arguments).

We have $N_{a b}^{R} \subseteq\left(N_{a b}^{R} \cap N_{[b c]}\right) \cup\left(N_{a b}^{R} \cap N_{[a c]}\right) \cup\{1\}$. By $a P^{m} b,\left|N_{a b}^{R}\right|>\left|N_{b a}^{R}\right|$. Thus, from (3) and (4) we obtain

$$
k_{[b c]}+k_{[a c]} \geq k_{[b c]}^{\prime}+k_{[a c]}^{\prime} .
$$

Similarly, we have $N_{b c}^{R} \subseteq\left(N_{b c}^{R} \cap N_{[a b]}\right) \cup\left(N_{b c}^{R} \cap N_{[a c]}\right) \cup\{1\}$. By $b P^{m} c,\left|N_{b c}^{R}\right|>$ $\left|N_{c b}^{R}\right|$. Thus, from (2) and (4) we obtain

$$
k_{[a b]}+k_{[a c]}^{\prime} \geq k_{[a b]}^{\prime}+k_{[a c]} .
$$


Summing up (5) and (6) yields

$$
k_{[a b]}+k_{[b c]} \geq k_{[a b]}^{\prime}+k_{[b c]}^{\prime} .
$$

Note that if (5) or (6) holds with strict inequality, then (7) holds with strict inequality.

We have $N_{a c}^{R} \subseteq\left(N_{a c}^{R} \cap N_{[a b]}\right) \cup\left(N_{a c}^{R} \cap N_{[b c]}\right) \cup\{1\}$. If $a P_{1} c$, then from (7) we obtain $\left|N_{a c}^{R}\right|>\left|N_{c a}^{R}\right|$ and $a P^{m} c$, the desired conclusion.

If $b P_{1} a$, then the inequality of (5) remains valid when adding two to the right hand side. Thus, by summing up this inequality and (6) we obtain $k_{[a b]}+k_{[b c]} \geq k_{[a b]}^{\prime}+k_{[b c]}^{\prime}+2$, which implies $\left|N_{a c}^{R}\right|>\left|N_{c a}^{R}\right|$ and $a P^{m} c$, the desired conclusion.

Similarly, if $c P_{1} b$, then the inequality of (6) remains valid when adding two to the right hand side. Thus, by summing up this inequality and (5) we again obtain $k_{[a b]}+k_{[b c]} \geq k_{[a b]}^{\prime}+k_{[b c]}^{\prime}+2$ and $a P^{m} c$, the desired conclusion. This exhausts all possible cases ${ }^{2}$ and $R^{m}$ is quasi-transitive on $\{a, b, c\}$ if $\mathcal{S}$ satisfies $(n-1) \mathrm{DP}$ for $\{a, b, c\}$.

Second, suppose that $\mathcal{S}$ satisfies $(n-2)$ EPDP for the triple $\{a, b, c\}$ and $\mathcal{S}$ does not satisfy $(n-1) \mathrm{DP}$ for $\{a, b, c\}$ (otherwise we use the previous proof to conclude that $R^{m}$ is quasi-transitive). Then $\mathcal{S}$ satisfies $(n-2) \mathrm{DP}$ and because $\mathcal{S}$ violates $(n-1) \mathrm{DP}$, we must have $\left|N_{\{a, b, c\}}^{f}\right|=2$. Without loss of generality, let $N_{\{a, b, c\}}^{f}=\{1,2\}$.

Note that $N_{a b}^{R} \backslash\{1,2\}=\left(N_{a b}^{R} \cap N_{[b c]}\right) \cup\left(N_{a b}^{R} \cap N_{[a c]}\right)$ and $N_{b a}^{R} \backslash\{1,2\}=$ $\left(N_{b a}^{R} \cap N_{[b c]}\right) \cup\left(N_{b a}^{R} \cap N_{[a c]}\right)$. By $a P^{m} b,\left|N_{a b}^{R}\right|>\left|N_{b a}^{R}\right|$. Thus, if $a P_{1} b$ and $a P_{2} b$, then $2+k_{[b c]}+k_{[a c]}>k_{[b c]}^{\prime}+k_{[a c]}^{\prime}$ and

$$
2+k_{[b c]}-k_{[b c]}^{\prime}>k_{[a c]}^{\prime}-k_{[a c]} .
$$

Note that $k_{[b c]}+k_{[b c]}^{\prime}=n_{[b c]}$. Thus, $k_{[b c]}-k_{[b c]}^{\prime}$ is even if $n_{[b c]}$ is even and $k_{[b c]}-k_{[b c]}^{\prime}$ is odd if $n_{[b c]}$ is odd. The same is true for $k_{[a c]}^{\prime}-k_{[a c]}$. Since $\mathcal{S}$ satisfies $(n-2)$ EPDP, $n_{[b c]}$ and $n_{[a c]}$ are either both even or both odd. Thus, in (8) the left hand side and the right hand side are either both even or both odd and the weak inequality remains true when adding two to the right hand side. Hence, if $a P_{1} b$ and $a P_{2} b$, then

$$
k_{[b c]}-k_{[b c]}^{\prime} \geq k_{[a c]}^{\prime}-k_{[a c]} .
$$

If $a P_{1} b$ and $b P_{2} a$ (or $b P_{1} a$ and $\left.a P_{1} b\right)$, then similarly we obtain

$$
k_{[b c]}-k_{[b c]}^{\prime} \geq k_{[a c]}^{\prime}-k_{[a c]}+2 .
$$

\footnotetext{
${ }^{2}$ If $c P_{1} a, a P_{1} b$, and $b P_{1} c$, then $R_{1}$ is not transitive, a contradiction.
} 
If $b P_{1} a$ and $b P_{2} a$, then similarly we obtain

$$
k_{[b c]}-k_{[b c]}^{\prime} \geq k_{[a c]}^{\prime}-k_{[a c]}+4 .
$$

Since $b P^{m} c$ we obtain analogously from $(n-2)$ EPDP the following inequalities: if $b P_{1} c$ and $b P_{2} c$, then

$$
k_{[a b]}-k_{[a b]}^{\prime} \geq k_{[a c]}-k_{[a c]}^{\prime} .
$$

If $b P_{1} c$ and $c P_{2} b$ (or $c P_{1} b$ and $b P_{1} c$ ), then similarly we obtain

$$
k_{[a b]}-k_{[a b]}^{\prime} \geq k_{[a c]}-k_{[a c]}^{\prime}+2 .
$$

If $c P_{1} b$ and $c P_{2} b$, then similarly we obtain

$$
k_{[a b]}-k_{[a b]}^{\prime} \geq k_{[a c]}-k_{[a c]}^{\prime}+4 .
$$

We now combine (9), (10), or (11) with (12), (13), or (14).

If (9) and (12) hold, then $a P_{1} c$ and $a P_{2} c$ and by taking the sum of the two inequalities, we have $k_{[a b]}+k_{[b c]} \geq k_{[a b]}^{\prime}+k_{[b c]}^{\prime}$. Hence, $\left|N_{a c}^{R}\right|>\left|N_{c a}^{R}\right|$ and $a P^{m} c$, the desired conclusion.

If (9) and (13) hold (or (10) and (12) hold), then $a P_{1} c$ (or $a P_{2} c$ ) and by taking the sum of the two inequalities, we have $k_{[a b]}+k_{[b c]} \geq k_{[a b]}^{\prime}+k_{[b c]}^{\prime}+2$. Hence, $\left|N_{a c}^{R}\right|>\left|N_{c a}^{R}\right|$ and $a P^{m} c$, the desired conclusion.

If (10) and (13) hold (or (11) or (14) holds), then by taking the sum of the two inequalities we obtain $k_{[a b]}+k_{[b c]} \geq k_{[a b]}^{\prime}+k_{[b c]}^{\prime}+4$, which implies $\left|N_{a c}^{R}\right|>\left|N_{c a}^{R}\right|$ and $a P^{m} c$, the desired conclusion.

Hence, $R^{m}$ is quasi-transitive on $\{a, b, c\}$ if $\mathcal{S}$ satisfies $(n-2)$ EPDP for $\{a, b, c\}$.

An immediate corollary of Theorem 1 is the characterization of the necessary and sufficient conditions for majority rule to be quasi-transitive when agents are allowed to have subjective indifferences (given their objective indifferences).

Definition $3(n-2)$ Unconcerned Agents $((n-2)$ UNCA). The partition profile $\mathcal{S}$ satisfies $(n-2)$ UNCA for a triple $\{a, b, c\}$ if at least $n-2$ agents cannot distinguish the alternatives belonging to the triple, i.e., there are distinct $j, k \in N$ such that for each $i \in N \backslash\{j, k\}$ there is some $S \in \mathcal{S}_{i}$ with $\{a, b, c\} \subseteq S$.

Corollary 1 Let $\mathcal{S}=\left(\mathcal{S}_{i}\right)_{i \in N}$. Then the majority rule is quasi-transitive on the domain $\mathcal{R}_{\mathcal{S}}$ if and only if $\mathcal{S}$ satisfies $(n-1)$ DP or $(n-2)$ UNCA for any triple of alternatives. 
Proof. We prove Corollary 1 along the lines of the proof of Theorem 1 . Throughout the proof we fix a triple $\{a, b, c\}$ of $A$. Let $N_{\{a, b, c\}}^{f}$ denote the set of agents for whom $\{a, b, c\}$ is free in $\mathcal{S}_{i}$.

(Only if) We show necessity. Suppose that majority rule is quasi-transitive on the domain $\mathcal{R}_{\mathcal{S}}$ and consider the triple $\{a, b, c\}$. Because $\mathcal{P}_{\mathcal{S}} \subseteq \mathcal{R}_{\mathcal{S}}$, we know by Theorem 1 that $\mathcal{S}$ satisfies $(n-1)$ DP or $(n-2)$ EPDP for $\{a, b, c\}$. Thus, we just need to show that if $\mathcal{S}$ satisfies $(n-2)$ EPDP and not $(n-1)$ DP for $\{a, b, c\}$, then $\mathcal{S}$ must satisfy $(n-2)$ UNCA for $\{a, b, c\}$. Suppose that $\mathcal{S}$ does not satisfy $(n-2)$ UNCA. Since $\mathcal{S}$ satisfies $(n-2)$ EPDP and not $(n-1)$ DP, we must have $\left|N_{\{a, b, c\}}^{f}\right|=2$. Since $\mathcal{S}$ satisfies $(n-2) \operatorname{EPDP}$ and violates $(n-2)$ UNCA, we have $N_{[a b]} \cup N_{[a c]} \cup N_{[b c]} \neq \emptyset$.

Without loss of generality, let $N_{\{a, b, c\}}^{f}=\{1,2\}$ and $i \in N_{[a c]}$. Then choose $R \in \mathcal{R}_{\mathcal{S}}$ such that

$$
\text { (1) } c P_{1} a P_{1} b ; \quad(2) a P_{2} b P_{2} c ; \quad \text { (3) } b P_{i} a I_{i} c
$$

and all other agents are indifferent among $a, b$, and $c$. Now by construction, $\left|N_{a b}^{R}\right|>\left|N_{b a}^{R}\right|$ and $a P^{m} b,\left|N_{b c}^{R}\right|>\left|N_{c b}^{R}\right|$ and $b P^{m} c$, and $\left|N_{a c}^{R}\right| \leq\left|N_{c a}^{R}\right|$ and $c R^{m} a$. Hence, majority rule is not quasi-transitive.

(If) We show sufficiency. If $\mathcal{S}$ satisfies $(n-1) \mathrm{DP}$ for $\{a, b, c\}$, then the proof is identical with the corresponding part of the proof of Theorem 1. If $\mathcal{S}$ satisfies $(n-2) \mathrm{UNCA}$ and not $(n-1) \mathrm{DP}$, then $\left|N_{\{a, b, c\}}^{f}\right|=2$ and $n_{[a b]}=n_{[a c]}=n_{[b c]}=0$. Thus, $\mathcal{S}$ satisfies $(n-2)$ EPDP for $\{a, b, c\}$ and by Theorem 1, majority rule is quasi-transitive.

Having proved our basic results, the rest of the section is devoted to introduce a number of relevant qualifications.

Example 1 Let $A=N$ and $\left\{N^{1}, N^{2}\right\}$ be a partition of $N$. For all $i \in N$, let $\mathcal{S}_{i}=\left\{\{i\}, N^{1} \backslash\{i\}, N^{2} \backslash\{i\}\right\}$. Let $\mathcal{S}=\left(\mathcal{S}_{i}\right)_{i \in N}$.

If $|N|=n$ is even, then it follows from Theorem 1 that majority rule is quasi-transitive on the domain $\mathcal{P}_{\mathcal{S}}$ : let $\{a, b, c\}$ be a triple of alternatives. If $\{a, b, c\} \subseteq N_{1}$ (or $\left.\{a, b, c\} \subseteq N_{2}\right)$, then $\mathcal{S}$ satisfies $(n-1)$ DP for $\{a, b, c\}$. Otherwise, one pair in the triple belongs to $N_{1}$ or $N_{2}$. If say $a$ and $b$ belong to $N_{1}$, then all agents in $N \backslash\{a, b\}$ regard $a$ and $b$ objectively indifferent (and distinguish $c$ from $a$ and $b$ ) and all numbers $n_{[a b]}=n-2, n_{[a c]}=0$, and $n_{[b c]}=0$ are even (since $n$ is even), i.e. $\mathcal{S}$ satisfies $(n-2)$ EPDP.

If $|N|=n$ is odd, then it follows from Theorem 1 that majority rule is not quasi-transitive on the domain $\mathcal{P}_{\mathcal{S}}$ : since $|N| \geq 3$, there exists a triple 
of alternatives $\{a, b, c\}$ such that $\left(a, b \in N^{1}\right.$ and $\left.c \in N^{2}\right)$ or $\left(c \in N^{1}\right.$ and $\left.a, b \in N^{2}\right)$. Then $\{a, b, c\}$ is a free triple for agents $a$ and $b$ and $\mathcal{S}$ violates $(n-1) \mathrm{DP}$ for the triple $\{a, b, c\}$. Furthermore, all agents in $N \backslash\{a, b\}$ regard $a$ and $b$ objectively indifferent and $n_{[a b]}=n-2$ is odd (since $n$ is odd) whereas $n_{[a c]}=0$ and $n_{[b c]}=0$ are even, i.e. $\mathcal{S}$ violates $(n-2)$ EPDP for the triple $\{a, b, c\}$.

Note that each agent's admissible preferences allow for at least one free triple (and therefore, for at least $n-2$ free triples) and often two agents can completely distinguish a given triple (more precisely, if $a$ and $b$ belong to $N^{1}$ and $c$ belongs to $N^{2}$, then $\{a, b, c\}$ is a free triple for $a$ and $b$ ).

Example 1 has the natural interpretation of a faculty board having to elect a department chair. Any faculty member is eligible, and usually the winner has the possibility of accepting or rejecting the election. Suppose the faculty is objectively divided into two groups, say theorists and applied, that each voter considers his or her own case as separate from the rest and that each voter treats all other candidates as members of one of the two groups, being indifferent between any two theorists or between any two applied people (other than his or her own). All possible orderings of the three personalized indifference classes (oneself, theorists and applied, minus eventually oneself) are admissible. For an even number of faculty members our conditions hold, and the use of the majority rule guarantees that the derived pairwise comparisons between candidates satisfy quasi-transitivity. If the number of faculty members is odd, then majority rule violates quasi-transitivity.

When the winner rejects the election, the chair is offered to the candidate who is the winner of the majority relation restricted to the remaining candidates. Hence, it is important that the majority relation yields for any set of alternatives a winner, i.e., majority rule is a social decision function. This is guaranteed by quasi-transitivity of the majority rule. ${ }^{3}$

Our next example is inspired by Dutta, Jackson, and Le Breton (2001) where a candidate has to be chosen for a position and both outside and inside candidates are considered for the position. The position could be the head of a subdivision in a company or an administrative position at a university. Then the set of inside candidates is the overlap between the set of alternatives and the set of voters. The outside candidates are the candidates who are not inside. Inside candidates distinguish between inside and outside candidates. Furthermore, the chair of the nomination committee (say the department head) and another representative of the institution (say the dean) are not

\footnotetext{
${ }^{3}$ Note that it is not guaranteed by acyclicity for three alternatives of the majority rule. See Sen and Pattanaik (1969, p. 199).
} 
candidates and can rank the candidates in any conceivable way, i.e. they can express strong preferences in their regard.

Example 2 Let $A \cap N \neq \emptyset$ and $|N \backslash A|=2$. We will call $A \cap N$ the set of inside candidates, $A \backslash N$ the set of outside candidates, and $N \backslash A$ the voters who are not up for nomination. For all $i \in A \cap N$, let $\mathcal{S}_{i}=\{A \cap N, A \backslash N\}$, and for all $i \in N \backslash A$, let $\mathcal{S}_{i}=\{\{a\} \mid a \in A\}$. Let $\mathcal{S}=\left(\mathcal{S}_{i}\right)_{i \in N}$.

If $n$ is even, then it follows from Theorem 1 that majority rule is quasitransitive on the domain $\mathcal{P}_{\mathcal{S}}$ : let $\{a, b, c\}$ be a triple of alternatives.

(i) If $\{a, b, c\} \subseteq A \cap N$ or $\{a, b, c\} \subseteq N \backslash A$, then all agents in $A \cap N$ rank $a, b$, and $c$ indifferent and $n_{[a b]}=n_{[a c]}=n_{[b c]}=0$. Hence, $\mathcal{S}$ satisfies $(n-2)$ EPDP for $\{a, b, c\}$.

(ii) If $a \in A \backslash N$ and $b, c \in A \cap N$, then $a$ is an outside candidate and no agent is indifferent between $a$ and $b$, i.e. $n_{[a b]}=0$. Similarly, $n_{[a c]}=0$. Furthermore, all agents in $A \cap N$ rank $b$ and $c$ indifferent and distinguish $a$ from $b$ and $c$, i.e. $n_{[b c]}=n-2$. Since $n$ is even, now all numbers $n_{[a b]}$, $n_{[a c]}$, and $n_{[b c]}$ are even and $\mathcal{S}$ satisfies $(n-2) \operatorname{EPDP}$ for $\{a, b, c\}$.

(iii) If $a, b \in A \backslash N$ and $c \in A \cap N$, then similarly as above it follows that $n_{[a b]}=n-2, n_{[a c]}=0$, and $n_{[b c]}=0$. Since $n$ is even, now all numbers $n_{[a b]}, n_{[a c]}$, and $n_{[b c]}$ are even and $\mathcal{S}$ satisfies $(n-2)$ EPDP for $\{a, b, c\}$.

Another application, where restrictions of the type we proposed do apply, is Demange (2004). She has studied the distribution of profits from cooperation in games with hierarchies. Hierarchies are described by a tree, which describes connections between agents, and by a specific individual, among all the agents, who plays the role of the principal. Demange (2004) shows that if only coalitions which are properly connected can form (she calls them teams), then the (restricted) core of the cooperative game among these agents is nonempty and easy to describe. The admissible teams (and thus, the resulting core distribution) depend on the tree and also on the specific agent who plays the role of principal. One may extend the analysis of Demange (2004) by separating these two ingredients, and by allowing all possible hierarchies which arise from the same tree, as the principal changes. In Demange's interpretation, the tree expresses possible channels of communication among agents. Suppose that these channels are technologically determined, but that the directions of hierarchical communication may be chosen. For example, all agents may vote on who is going to play the role of the principal. Their preferences will depend on the payoffs that they will get in the core, depending on who is the principal (for a given tree). It turns out 
that agents will get the same payoff for all principals who are on the same branch away from some of their immediate neighbors. That is, preferences induced by the proposed extension of Demange's model satisfy $(n-1)$ DP: given a triple $\{a, b, c\}$, there is exactly one agent for whom $\{a, b, c\}$ is free, namely the agent who is located at the median of $a, b$ and $c$. Hence, the majority rule would always determine (at least) one winner if agents in that context would vote for a principal. This example is mentioned to illustrate that the restriction arises even in rather unexpected contexts. It suggests that other situations where someone must be chosen to play a special role, may give rise to similar conditions. Of course, voting is only one of the possible methods to choose an agent to play a role. In certain contexts, especially if side payments are possible, these roles may be auctioned (Pérez-Castrillo and Wettstein, 2002). But voting is, to say the least, one of the most prevailing methods to choose agents, and it is good to know about conditions where its simplest version, simple majority, will work properly.

\section{Related Literature}

Note that all our properties could have been equivalently defined for preference profiles instead for the partition profile $\mathcal{S}$ : for instance, just replace "for some $S \in \mathcal{S}_{i},\{a, b\} \subseteq S$ " by " $a I_{i} b$ ". We chose to state them in terms of $\mathcal{S}$ because then the definitions are independent of whether we consider the set of strict profiles or the set of weak profiles admissible by $\mathcal{S}$. Below we will refer to the equivalent definition of our properties for preference profiles when comparing them with restrictions on preference profiles made by previous literature.

There exists a vast literature on domain restrictions and their implications under different rules of preference aggregation. A very complete monograph is due to Gaertner (2001). The most studied aggregation rule is simple majority, and the standard properties which are sought from the majority relation are transitivity, quasi-transitivity, acyclicity or the existence of a maximal element of the relation. Among the many restrictions which have been studied, the most popular is still that of single-peakedness (Black, 1948). Other domains were analyzed by Inada (1964,1969), Sen and Pattanaik (1969), Demange (1982), and Grandmont (1978). ${ }^{4}$ Our domains are different than any of those we just mentioned, and they do not seem to have been considered by the previous literature. We now comment briefly on the analogies and

\footnotetext{
${ }^{4}$ Inada $(1964,1969)$ and Grandmont $(1978)$ were concerned with the transitivity of the majority relation, Sen and Pattanaik (1969) with the quasi-transitivity of the majority relation, and Demange (1982) with the existence of a maximal element.
} 
the difference between our type of restrictions and those proposed by other authors. There is a fundamental difference between our setup and all the others we mention (with the exception of Grandmont's). In our case, the set of orders of the alternatives which are admissible may be different for each of the agents. Indeed, each voter is allowed to have at most as many indifference classes as the number of members in his partition of objective indifference classes. Hence, agents are allowed to have different sets of preferences. Under our restrictions, admissible domains are personalized. By contrast, the classical restrictions we now briefly review do limit the set of preferences which are admissible, but then allow all agents to exhibit any of the preferences in this common pool. Inada (1964) considered the case where each agent can classify the set of alternatives into two groups, and then will consider all alternatives within the same group as indifferent. He was concerned with transitivity of the majority rule and showed that if a profile satisfies $n$ DP (for each agent there is a pair which he regards indifferent), then majority rule is transitive. Obviously $n$ DP implies $(n-1)$ DP but not vice versa. Furthermore, note that Inada (1964) deals with transitivity of majority rule whereas we deal with quasi-transitivity and Inada (1964) did not search for necessary conditions. Our conditions also rely on the establishment of "large" indifference classes, but the analogy stops here.

Another interesting set of restrictions were proposed by Sen and Pattanaik (1969) and Inada (1969). Let $R \in \mathcal{R}^{N}$ and $\{a, b, c\}$ be a triple of alternatives. The profile $R$ satisfies value restriction (VR) for the triple $\{a, b, c\}$ if there is one alternative in the triple, say $a$, that is not ranked worst (or best or medium) by all individuals who are not indifferent between $a, b$, and $c$ (i.e. (for all $i \in N$ such that $\neg a I_{i} b I_{i} c, a P_{i} b \vee a P_{i} c$ ) or (for all $i \in N$ such that $\left.\neg a I_{i} b I_{i} c, b P_{i} a \vee c P_{i} a\right)$ or (for all $i \in N$ such that $\neg a I_{i} b I_{i} c,\left(a P_{i} b \wedge a P_{i} c\right) \vee$ $\left.\left.\left(b P_{i} a \wedge c P_{i} a\right)\right)\right)$. The profile $R$ satisfies extremal restriction (ER) for the triple $\{a, b, c\}$ if the following holds: if $b P_{i} a P_{i} c$ for some $i \in N$, then $c P_{j} a P_{j} b$ for all $j \in N$ such that $c P_{j} b$. The profile $R$ satisfies limited agreement (LA) for the triple $\{a, b, c\}$ if there are two alternatives, say $b$ and $c$, such that $b R_{i} c$ for all $i \in N$.

These restrictions define domains under which the majority rule and other forms of binary comparisons will be well behaved. Sen and Pattanaik (1969, Theorem V) show that a necessary and sufficient condition for the majority rule to be quasi-transitive is that a profile satisfies for each triple of alternatives at least one of the conditions, VR, ER or LA. However, in their result the number of individuals is variable and therefore it does not apply to our model. Sen and Pattanaik (1969, p. 192) show that any profile violating VR, $\mathrm{ER}$, and LA for a triple $\{a, b, c\}$ must include a three-ordering-subset of the 
form $[1.1,2.1$ or 2.2 or $2.3,3.1$ or 3.2 or 3.3$]$ :

$$
\begin{array}{llllll}
(1.1) & a P_{i} b P_{i} c & & & & \\
(2.1) & b P_{i} c P_{i} a & (2.2) & b P_{i} c I_{i} a & (2.3) & b I_{i} c P_{i} a \\
(3.1) & c P_{i} a P_{i} b & (3.2) & c P_{i} a I_{i} b & (3.3) & c I_{i} a P_{i} b
\end{array}
$$

Note that when $n$ is even, $(n-1)$ DP and $(n-2)$ EPDP only rule out $[1.1,2.1,3.1]$. Any other combination is allowed by $(n-1)$ DP and $(n-2)$ EPDP. If $n$ is odd, say $n=5$, then $(n-1)$ DP and $(n-2)$ EPDP allow for [1.1,2.1 and 2.3,3.2 and 3.3]. Furthermore, by Theorem 1, none of Sen and Pattanaik's (1969) conditions is necessary in our model.

Demange (1982) proposed an extension of single-peakedness based on the relative positions of alternatives in the vertices of a tree (the original notion of single-peakedness is based on their position on a line, which is a very special tree). ${ }^{5}$ Demange's proposal bears a resemblance with ours in that it builds from a set of a priori given connections among alternatives which can be formalized as a tree. Finally, Grandmont (1978) proposed a notion of intermediate preferences leading to attractive and quite different domain restrictions. This notion is based upon the possibility of defining when an agent is in between two others. It requires that, if the two initial agents agree on how to rank a certain subset of alternatives, than any agent who is intermediate between them also shares these common preferences.

\section{References}

Black, D. (1948): "On the Rationale of Group Decision-making," Journal of Political Economy 56, 23-34.

Bogomolnaia, A., H. Moulin, and R. Stong (2005): "Collective Choice under Dichotomous Preferences," Journal of Economic Theory 122, 165-184.

Demange, G. (1982): "Single-Peaked Orders on a Tree," Mathematical Social Sciences 3, 389-396.

Demange, G. (2004): "On group stability in hierarchies and networks," Journal of Political Economy 112, 754-778.

Dutta, B., M.O. Jackson, and M. Le Breton (2001): "Strategic Candidacy and Voting Procedures," Econometrica 69, 1013-1037.

\footnotetext{
${ }^{5} \mathrm{~A}$ preference relation on a tree is a single-peaked order if it's restriction to any path of the tree is single-peaked.
} 
Gaertner, W. (2001): Domain Conditions in Social Choice Theory, Cambridge University Press.

Grandmont, J.-M. (1978): "Intermediate Preferences and the Majority Rule," Econometrica 46, 317-330.

Inada, K.-I. (1964): "A Note on the Simple Majority Decision Rule," Econometrica 32, 525-531.

Inada, K.-I. (1969): "The Simple Majority Decision Rule," Econometrica 37, 490-506.

Pattanaik, P.K., and A.K. Sen (1969): "Necessary and Sufficient Conditions for Rational Choice under Majority Decision," Journal of Economic Theory 1, 178-202.

Pérez-Castrillo, J.D., and David Wettstein (2002): "Choosing Wisely: A Multi-Bidding Approach," American Economic Review 92, 1577-1587.

Plott, C.R. (1973): "Path Independence, Rationality, and Social Choice," Econometrica 41, 1075-1091. 